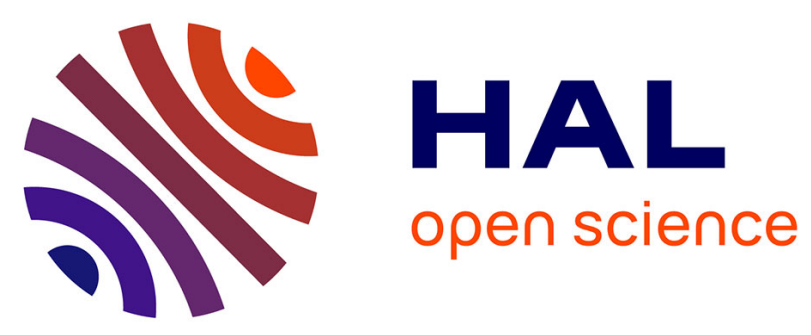

\title{
Patterns of proton therapy use in pediatric cancer management in 2016: An international survey
}

Neige Journy, Daniel J. Indelicato, Diana R. Withrow, Tetsuo Akimoto, Claire Alapetite, Masayuki Araya, Andrew Chang, John Han-Chih Chang, Brian Chon, Michael E. Confer, et al.

\section{To cite this version:}

Neige Journy, Daniel J. Indelicato, Diana R. Withrow, Tetsuo Akimoto, Claire Alapetite, et al.. Patterns of proton therapy use in pediatric cancer management in 2016: An international survey. Radiotherapy \& Oncology, 2019, 132, pp.155 - 161. 10.1016/j.radonc.2018.10.022 . hal-03486947

\section{HAL Id: hal-03486947 https://hal.science/hal-03486947}

Submitted on 20 Dec 2021

HAL is a multi-disciplinary open access archive for the deposit and dissemination of scientific research documents, whether they are published or not. The documents may come from teaching and research institutions in France or abroad, or from public or private research centers.
L'archive ouverte pluridisciplinaire HAL, est destinée au dépôt et à la diffusion de documents scientifiques de niveau recherche, publiés ou non, émanant des établissements d'enseignement et de recherche français ou étrangers, des laboratoires publics ou privés.

\section{(ㄷ)(1) $\$$}

Distributed under a Creative Commons Attribution - NonCommerciall 4.0 International 
Version of Record: https://www.sciencedirect.com/science/article/pii/S0167814018335461

Manuscript_0e780b015d797ea5a5010e1b6f908d7d

Patterns of proton therapy use in pediatric cancer management in 2016: an international survey

Neige Journy, PhD, INSERM U1018, Centre for Research in Epidemiology and Population Health, Cancer \& Radiations group, Gustave Roussy Cancer Campus, B2M, 39 rue Camille Desmoulins, 94800 Villejuif, France

Daniel J. Indelicato, MD, University of Florida Department of Radiation Oncology, 2015 N. Jefferson St., Jacksonville, FL 32206, United States

Diana R. Withrow, PhD, Radiation Epidemiology Branch, Division of Cancer Epidemiology and Genetics, National Cancer Institute, National Institutes of Health, 9609 Medical Center Drive, MSC 9778, Bethesda, MD 20892, United States

Tetsuo Akimoto, MD, National Cancer Center Hospital East, 6-5-1 Kashiwanoha, Kashiwa, Chiba 277-8577, Japan

Claire Alapetite, MD, Institut Curie Proton therapie Centre (ICPO), Campus universitaire, 15 Rue Georges Clemenceau, 91400 Orsay, France

Masayuki Araya, Aizawa Hospital Proton Therapy Center, 2-5-1 Honjou Matsumoto City, Nagano 390-8510, Japan

Andrew Chang, MD, California Protons, 9730 Summers Ridge Road, San Diego, CA 92121, United States

John Han-Chih Chang, MD, Northwestern Medicine Chicago Proton Center, 4455 Weaver Parkway, Warrenville, IL 60555, United States; Vanderbilt University Medical Center, Department of Radiation Oncology, 2220 Pierce Ave, Nashville, TN 37232, United States

Brian Chon, MD, ProCure Proton Therapy Center, 103 Cedar Grove Lane Somerset, NJ 08873, United States 
Michael E. Confer, MD, ProCure Proton Therapy Center, 5901 West Memorial Road, Oklahoma City, OK 73142, United States

Yusuke Demizu, MD, Hyogo Ion Beam Medical Center, 1-2-1 Kouto, Shingu-cho, Tatsuno, Hyogo 679-5165, Japan

Rémi Dendale, MD, Institut Curie Proton therapie Centre (ICPO), Campus universitaire, 15 Rue Georges Clemenceau, 91400 Orsay, France

Jérôme Doyen, MD, Department of Radiation Oncology, Centre Antoine-Lacassagne, 227 avenue de la Lanterne, University of Côte d'Azur, 06000 Nice, France

Ralph Ermoian, MD, SCCA Proton Therapy, 1570 N 115th St, Seattle, WA 98133, United States

Kristin Gurtner, MD, Universitäts Protonentherapie Dresden, Universitätsklinikum Carl Gustav Carus, Fetscherstrasse 74, 01307 Dresden, Germany

Christine Hill-Kayser, MD, Children's Hospital of Philadelphia and University of Pennsylvania Roberts Proton Therapy Center), 3400 Civic Center Blvd, Philadelphia, PA 19104, United States

Hiromitsu Iwata, MD, Nagoya Proton Therapy Center (Nagoya City West Medical Center), 462-8508, 1-1-1 Hirate-cho, Kita-ku, Nagoya-city, Japan

Joo-Young Kim, MD, ProtonTherapy Center, National Cancer Center, 323, Ilsan-ro, Ilsandong-gu, Goyang-si, Gyonggi-do 10408, South Korea

Young Kwok, MD, Departement of Radiation Oncology, University of Maryland School of Medicine, 22 South Greene St, Baltimore, MD 21201, United States

Nadia N. Laack, MD, Department of Radiation Oncology, Mayo Clinic, 200 1st St SW, Rochester, MN 55902, United States 
Choonsik Lee, PhD, Radiation Epidemiology Branch, Division of Cancer Epidemiology and Genetics, National Cancer Institute, National Institutes of Health, 9609 Medical Center Drive, MSC 9778, Bethesda, MD 20892, United States

Do Hoon Lim, MD, Samsung Proton Therapy Center, Samsung Medical Center, Sungkyunkwan University School of Medicine, 81 Irwon-ro Gangnam-gu, Seoul 06351, South Korea

Lilia Loredo, MD, Loma Linda University Medical Center, 11235 Anderson St Loma Linda, CA 92354, United States

Victor S. Mangona, MD, Texas Center for Proton Therapy, 1501 W Royal Ln, Irving, TX 75063, United States

David B. Mansur, MD, University Hospitals Proton Therapy Center, Seidman Cancer Center, Rainbow Babies and Children's Hospital, Case Western Reserve University, 11100 Euclid Avenue, Cleveland, OH 44106, United States

Masao Murakami, MD, Southern TOHOKU Proton Therapy Center, 172-7choume, Yatsuyamada, Koriyama, Fukushima 963-8563, Japan

Shigeyuki Murayama, MD, Shizuoka Cancer Center, 1007 Shimonagakubo, Nagaizumi-cho, Suntougun, Shizuoka 411-8777, Japan

Takashi Ogino, MD, Medipolis Proton Therapy and Research Center, 4423 Higashikata Ibusuki, Kaghosima 890-0304, Japan

Barbora Ondrová, MD, Proton Therapy Center Czech s.r.o., Budínova 1a, 18000 Praha, Czech Republic

Rahul R. Parikh, MD, Rutgers Cancer Institute of New Jersey, 195 Little Albany St, New Brunswick, NJ 08903, United States 
Arnold C. Paulino, MD, The University of Texas M.D Anderson Cancer Center, Department of Radiation Oncology, 1515 Holcombe Blvd, Box 97, Houston, TX 77030, United States

Stephanie Perkins, MD, S. Lee Kling Proton Therapy Center, Washington University in St. Louis, 4921 Parkview Place, Saint Louis, MO 63110, United States

Naren R. Ramakrishna, MD, Orlando Health UF Health Cancer Center, 1400 S. Orange Ave, Orlando, FL 32806, United States

Ronald Richter, MD, Rinecker Proton Therapy Center, Schaeftlarnstr. 133, 81371 Muenchen, Germany

Barbara Rombi, MD, Proton Therapy Center, Via Al Desert 14, 38122 Trento, Italy

Satoshi Shibata, MD, Proton Therapy Center, Fukui Prefectural Hospital, 2-8-1 Yotsui, Fukui, Fukui 910-8526, Japan

Shinichi Shimizu, Department of Radiation Oncology, Hokkaido University Faculty of Medicine, 060-8638 Hokkaido, Sapporo, Kita-ku, Kita 15, Nishi 7, Japan

Beate Timmermann, MD, Department of Particle Therapy, University Hospital Essen, West German Proton Therapy Center Essen (WPE), West German Cancer Center (WTZ), German Cancer Consortium (DKTK), Hufelandstr. 55, 45147 Essen, Germany

Tamara Vern-Gross, DO, Mayo Clinic Arizona, 5881 E Mayo Blvd, Phoenix, AZ 85054, United States

Chiachien J. Wang, MD, Willis-Knighton Proton Center, 2600 Kings Highway, Shreveport, LA 71103, United States

Damien C. Weber, MD, Center for Proton Therapy, Paul Scherrer Institute, 5232 Villigen PSI West, ETH Domain, Switzerland 
J. Ben Wilkinson, MD, Provision Cares Proton Therapy Center, 6450 Provision Cares Way, Knoxville, TN 37909, United States

Petra Witt Nyström, MD, The Skandion Clinic, von Kraemers allé 26, 75237 Uppsala, Sweden; Danish Centre for Particle Therapy, Palle Juul-Jensens Blvd. 99, 8200 Aarhus, Denmark

Torunn I. Yock, MD, Francis H Burr Proton Therapy Center, Massachusetts General Hospital, 30 Fruit Street, Boston, MA 02114, United States

Ruth A. Kleinerman, PhD, Radiation Epidemiology Branch, Division of Cancer Epidemiology and Genetics, National Cancer Institute, National Institutes of Health, 9609 Medical Center Drive, MSC 9778, Bethesda, MD 20892, United States

Amy Berrington de Gonzalez, DPhil, Radiation Epidemiology Branch, Division of Cancer Epidemiology and Genetics, National Cancer Institute, National Institutes of Health, 9609 Medical Center Drive, MSC 9778, Bethesda, MD 20892, United States

Corresponding author: Neige Journy, INSERM U1018, Centre for Research in Epidemiology and Population Health, Cancer \& Radiations group, Gustave Roussy Cancer Campus, B2M, 39 rue Camille Desmoulins, 94800 Villejuif, France. E-mail: neige.journy@gustaveroussy.fr

\section{Sources of funding}

This work was supported by the National Institutes of Health Intramural Research Program, and the Fondation ARC pour la recherche sur le cancer (grant nPDF20161205256, N. Journy). The sponsors had no role in study design, in the collection, analysis and interpretation of data, in the writing of the report, and in the decision to submit the article for publication. 


\section{ABSTRACT}

Purpose: To facilitate the initiation of observational studies on late effects of proton therapy in pediatric patients, we report on current patterns of proton therapy use worldwide in patients aged less than 22 years.

Materials \& Methods: Fifty-four proton centers treating pediatric patients in 2016 in 11 countries were invited to respond to a survey about the number of patients treated during that year by age group, intent of treatment, delivery technique and tumor types.

Results: Among the 40 participating centers (participation rate: $74 \%$ ), a total of 1,860 patients were treated in 2016 (North America: 1,205, Europe: 432, Asia: 223). The numbers of patients per center ranged from 1 to 206 (median: 29). Twenty-four percent of the patients were <5 years of age, and $50 \%<10$ years. More than 30 pediatric tumor types were identified, mainly treated with curative intent: $48 \%$ were CNS, 25\% extra-cranial sarcomas, $7 \%$ neuroblastoma, and 5\% hematopoietic tumors. About half of the patients were treated with pencil beam scanning. Treatment patterns were broadly similar across the three continents.

Conclusion: To our knowledge, this survey provides the first worldwide assessment of proton therapy use for pediatric cancer management. Since previous estimates in the United States and Europe, CNS tumors remain the cancer types most commonly treated with protons in 2016. However, the proportion of extra-cranial tumors is growing worldwide. The typically low numbers of patients treated in each center indicate the need for international research collaborations to assess long-term outcomes of proton therapy in pediatric patients.

KEY-WORDS: Proton therapy, Paediatrics, Patterns of care, Survey 


\section{INTRODUCTION}

Several national guidelines consider proton therapy an optimal radiation modality for treating pediatric tumors and reducing treatment toxicities [1-6]. Compared with photons, protons have better physical properties providing clear dosimetric advantages to improve treatment conformality and lower doses to surrounding normal tissues [7-9]. This could have considerable clinical benefits to reduce treatment toxicities while maintaining or improving cure rates, especially when treating young patients with a tumor located close to critical normal tissues, such as the brain stem, eyes and spinal cord . Over the past decade, an expanding number of clinical outcome studies on proton therapy has been provided [9]. However, little data on late effects is yet available. Few phase-II [10, 11] and, to our knowledge, no phase-III [12], randomized controlled trials have been conducted to compare clinical outcomes of proton vs. photon therapy in the pediatric setting. Observational data on late effects also remain sparse due to small sample sizes in single-institution studies and limited follow-up capturing long-term outcomes [13-16]. Despite the limited information on late effects, the demonstrated dosimetric advantages of protons have led to increasing use of this technique. Since 2012, the number of proton centers has increased from 34 to 61 worldwide [17]. By 2025, 132 centers are planned to be operating in 31 countries, and the numbers of patients treated with protons are expected to rapidly increase in the next coming years. Assessing the long-term clinical benefits and toxicities of proton therapy is thus critically needed.

In addition to the United States (U.S.) Pediatric Proton Consortium Registry which currently involves 13 centers across the country [18], an International Pediatric Proton Therapy Consortium has been proposed to build cooperation across institutions and initiate international collaborative studies evaluating long-term effects [19]. As a first step of this initiative, we conducted a patterns of care survey to estimate the number of pediatric patients treated with protons in 2016 and describe the clinical indications and delivery techniques used worldwide.

\section{MATERIALS \& METHODS}


The survey was conducted between July 2017 and June 2018 in all proton centers treating pediatric patients in 2016 worldwide. A total of 61 centers operating in 16 countries were identified through the Particle Therapy Co-Operative Group (PTCOG) website [17]. After exclusion of eye proton therapy $(n=5)$ and adult cancer $(n=1)$ centers and another one that stopped treating patients before 2016, 54 centers located in 11 countries were eligible for the survey. We invited all eligible centers to participate.

The questionnaire was adapted from the U.S. 2010-2012 survey [20] to collect information about the number of patients $<22$ years of age treated in 2016 at each center, by age group $(<1,1$ to $<5,5$ to $<10,10$ to $<15$, and 15 to $<22$ years), intent of treatment (curative, palliative), delivery modality (primary or boost irradiation) and technique (passive scattering, uniform scanning, or pencil beam scanning), tumor types or non-malignant diseases (e.g. arteriovenous malformation), and patients' country of residency. The survey did not request any personal identifying information, and was exempt from IRB review per 45 CFR 46 and NIH Policy by the NCI Office of Human Research Protections Program. The questionnaire was sent to key contacts identified through multiple sources including the PTCOG website, national cancer organizations, authors of peer-reviewed publications or the center's website. The survey was closed on June 25, 2018. Descriptive statistics were computed with SAS 9.4 (SAS Institute Inc., Cary, NC, United States). Percentage ranges across centers are provided only among those with $\geq 10$ patients, while other statistics include all participating centers.

\section{RESULTS}

\section{Number of treated patients}

A total of 40 centers participated in the survey, including 20 in North America, nine in Europe and 11 in Asia (Table 1). Among the 54 eligible centers treating pediatric patients in 2016, the participation rate was $74 \%$ overall. The non-participating centers did not respond $(n=12)$ or declined $(n=2)$ the invitation. In the 40 participating centers, 1,860 patients $<22$ years of age were treated with protons in 
2016: 1,205 in North America, 432 in Europe and 223 in Asia. Ten (25\%) centers treated <10 patients, while $19(48 \%)$ treated $\geq 30$ patients and $7(18 \%) \geq 100$ patients. Six out of the seven centers treating $\geq 100$ patients were located in North America. In centers that started treating patients before 2016, the median number of patients per center was 29 ( $n=36$, range: 1 to 206) overall, 68 ( $n=16$, range: 1 to 206) in North America, $31(\mathrm{n}=9$, range: 2 to 171) in Europe and $12(\mathrm{n}=11$, range: 2 to 62$)$ in Asia. By center, no association was observed between the number of patients in 2016 and the center's first year of operation (Figure 1).

\section{Intent of treatment, delivery modality, and technique}

The intent of treatment was curative in $98 \%$ of patients in all continents (range across centers: 86 to $100 \%$ ). Information on this question was available for $1788 / 1860$ patients treated in $38 / 40$ centers. Protons were used as boost irradiation in $2 \%$ of patients (range across centers: 0 to $20 \%$ ). Pencil beam scanning was available in $25(63 \%)$ centers, passive scattering in $16(40 \%)$, and uniform scanning beam in ten (25\%); 11 (28\%) centers having $\geq 2$ techniques available. Pencil beam scanning was used in $13(65 \%)$ centers located in North America, seven (78\%) in Europe, and five (45\%) in Asia. A total of $950(51 \%)$ of the pediatric patients were treated with pencil beam scanning (Table 2).

\section{Patient age}

Overall, $923(50 \%)$ patients were $<10$ years old, and $446(24 \%)$ were $<5$ years. The proportion of pediatric patients $<10$ years was slightly higher in Europe $(58 \%)$ and Asia $(61 \%)$ than in the U.S. (44\%) (Figure A1). In all continents, the proportion of children $<10$ years was lower in centers that opened most recently: $47 \%$ (range across centers: $33 \%$ to $68 \%$ ) in the 28 centers that opened in 20052015 compared to $64 \%$ (range across centers: $53 \%$ to 95\%) in the eight centers that opened <2005 (Figure A2).

\section{Types of pediatric diseases treated}


Forty-eight percent of the patients were treated for CNS tumors, with medulloblastoma, ependymoma, low-grade glioma, and craniopharyngioma being the most frequent tumor types (Table 3). An additional $14 \%$ of patients were treated for other head and neck tumors. Extra-cranial sarcomas accounted for $25 \%$ of the patients, with similar frequencies in all continents. In Europe and Asia, the proportion of head and neck tumors treated was higher in centers that opened more recently than in the centers that have been opened longer (Figure A3). The opposite trend was observed in North America where the proportion of head and neck tumors decreased, but the proportion of hematopoietic tumors increased, with the center's first year operating. Medulloblastoma, rhabdomyosarcoma, Hodgkin lymphoma (in North America), neuroblastoma (in North America and Asia), ependymoma and Ewing sarcoma were treated in $>70 \%$ to $100 \%$ of centers, while other tumor types were treated in fewer centers (Figure 2).

\section{DISCUSSION}

This first survey of worldwide patterns of proton therapy use for pediatric cancer management included 40 centers located in 11 countries. With a participation rate of $74 \%$, we estimate that between 2,000 and 2,500 pediatric patients were treated with protons in 2016. Pediatric cancers represented an estimated $10 \%$ of all proton therapy patients treated in 2016 [17]. In countries where information was available regarding the total number of pediatric patients receiving radiotherapy, the percentage of them treated with protons (either as primary treatment or boost irradiation) was $10 \%$ in France [21] and Japan [22], 15\% in the U.S. [20], and 33\% in Sweden (Dr. Petra Witt Nyström, emailed personal communication, 2018 June 18). In the U.S., we estimate that, for some agressive tumor types (e.g. rhabdomyosarcoma, medulloblastoma, and ependymoma), protons are currently used to treat up to $50-70 \%$ of all pediatric patients receiving radiotherapy (Table 4).

Our data suggest that the number of patients aged $<15$ years treated with protons has doubled in the U.S. between 2012 [20] and 2016. This trend reflects the substantial increase in the number of centers treating pediatric patients in the U.S., from nine in 2012 to 23 in 2016, and increased acceptance of 
proton therapy's role in pediatric oncology. In Europe, the number of patients aged $<18$ years has increased even more rapidly, by $50 \%$ since 2014 when 7 centers were in operation [23] (vs. 12 centers in 2016).

The number of pediatric patients varies widely across centers, with $25 \%$ of centers treating less than 10 patients per year and $18 \%$ of centers treating $\geq 100$ patients per year currently. The annual number of patients per center was often much lower in Asia (median: 12) and in Europe (median: 31) compared to North America (median: 68), but with a wide range between the centers. Several factors may influence the pediatric volume in proton centers. These include the technical capability (type of proton facility, technique of proton delivery, number of gantries) and the local organization of pediatric care, especially the presence and work time of pediatric radiation oncologists and anesthetists. The lower median number of treated patients per center in Asia and Europe may also reflect a lower propensity of pediatric medical or radiation oncologists to refer their patients to or use protons in those regions, a more limited access and referral capabilities to proton centers (including travel burden for the families), barriers for collaboration between referral pediatric oncology departments and proton centers, and/or lower financial incentives or pressure for proton therapy use in countries with national health insurance schemes, although all these issues also arise in the U.S. The number of patients referred from foreign countries, however, did not vary between the continents (Table 1), and the number of patients treated in 2016 was not clearly related to the centers' first operating year (Figure 1).

The variable pediatric volume across radiotherapy centers raises the question of whether there is sufficient expertise and experience in specific clinical indications to ensure an acceptable level of quality of care. This might be especially true in the specific context of modern precision therapies for pediatric cancer management, because these involve high technical complexity and unique considerations of treating a child with radiation, especially in terms of late effects. This issue has real implications, as systematic reviews from both Europe [24] and the U.S. [25] have demonstrated better 
survival among children with brain tumors and sarcomas treated at high volume centers. To address this, some countries, such as Denmark, the Netherlands, and Sweden actively use centralized medical systems to concentrate pediatric cancer patients in high volume proton/photon centers with pediatric radiotherapy expertise. In North America and Germany, some proton centers serve a similar role and concentrate a large number of pediatric patients. Nonetheless, volume activity requirements need to be considered in relation to accessibility of care, and the optimal balance between the two is definitely not obvious, especially in large countries. Selected academic medical centers thus offer additional training in pediatric radiation oncology targeting the whole radiation oncology community with a focus on the technical specifics of proton therapy. As more and more proton centers come online, credentialing criteria or standards potentially including a minimal pediatric volume activity requirement may be established in addition to training for centers and physicians intending on treating children.

In our survey, a very wide variety of pediatric tumor types was treated with protons. More than 30 tumor types were identified, the ten most frequent accounting for $75 \%$ of patients (Table 3 ). Since previous surveys carried out in the U.S. [20] and in Europe [23], CNS tumors remain the most commonly treated tumor types in 2016. However, with a wider variety of tumors treated, the increased technical capability of large "gantry-based" systems, and advanced treatment planning techniques to improve sparing of organ at risks, the proportion of all proton-treated cancers that are CNS tumors declined in the U.S. from $62 \%$ in 2010 to 55\% in 2012 [20], and $48 \%$ in 2016 (considering CNS histologies of the International Classification of Childhood Cancers as listed in Table 3). In Europe, the median proportion across centers of extracranial tumors increased from 17\% in 2014 [23] to $27 \%$ in 2016 , as well as the proportion of medulloblastoma which became one of the two leading indications for pediatric proton therapy in 2016 (Table 3).

Our findings regarding tumor types broadly reflect national guidelines and informal priorities for clinical indications for proton therapy in pediatrics [1-6]. General consensus was observed across 
centers to consider many pediatric tumors, including but not limited to rhabdomyosarcoma, medulloblastoma, ependymoma, low-grade glioma, craniopharyngioma, intracranial germ-cell tumor and Ewing sarcoma, as appropriate clinical indications for proton therapy, with $>70 \%$ of centers treating those tumor types in 2016 (Figure 2). There was emerging consensus across centers in North America and Asia to treat patients with neuroblastoma, but less clarity in Europe. We estimate that one in three patients treated with radiotherapy for neuroblastoma in the U.S. currently receives protons (Table 4). The most notable differences between the continents was for skull base chordoma/chondrosarcoma (60-70\% of centers in North America and Europe treating this tumor type vs. $0 \%$ in Asia) and Hodgkin lymphoma (>80\% of centers in North America treating this tumor vs. $15 \%$ in Asia and Europe). These indications (and variation) in the application of proton therapy are largely consistent with the conclusions of an international expert consensus panel convened in 2016 $[8]$.

Different proton delivery techniques were used across the countries in 2016. In the U.S. and Europe, pencil beam scanning was available at most centers (20/29 centers) and used to treat more than half of proton-treated patients (Table 2). In Asia, fewer centers (5/11 centers) used this technique, and half of the patients were treated with passive scattering and one-fifth with uniform scanning beam. The different dose distribution of each technique may have important implications in terms of tumor control and toxicity rates. Better proximal and distal dose conformality with pencil beam scanning may reduce doses to surrounding normal tissues, but passive scattered techniques provide more robust dose distribution currently for moving targets. Moreover, aperture-based passive scattering techniques result in a tighter radiation penumbra and thus reduce dose lateral to the target compared to pencil beam scanning that does not use apertures [26]. In contrast, the whole-body secondary neutron dose is increased with the use of apertures. The effect of proton delivery techniques on normal tissue exposures and toxicity risks thus remains controversial. The high potential for neutrons to induce chromosomal aberrations and DNA damages $[27,28]$ and the variations in proton and neutron dose 
distribution with different proton beam quality, incidence and collimation techniques $[26,29,30]$ may substantially affect the risk of late effects. Simulation studies have predicted reduced risks of second malignancies with pencil beam scanning [26], but those results should be interpreted cautiously and as illustrative only of the various physical dose distributions with different radiotherapy techniques as long as large uncertainties remain on the biological effects of neutron doses [27, 28].

The present survey has two main limitations. First, despite our efforts to reach all centers, the survey did not reach $100 \%$ participation rate in all countries, with 14 out of the 54 non-adult centers not responding to the questionnaire. However, it is possible that some of the non-respondents did not treat pediatric patients in 2016. Second, the survey collected no individual data to avoid requiring ethical agreements for each center and increase the participation rate, which prevented us from assessing the relationship between individual characteristics and identifying specific tumor types for which protons were used as boost irradiation or re-irradiation.

Nonetheless, the survey highlights important considerations for patient care. First, evidence suggests that pediatric proton therapy is still concentrated among relatively few centers, which has implications for training and workforce expertise distribution. Second, proton therapy represents a sizable financial investment. In planning a facility, design and staffing should incorporate age-specific considerations encompassing anesthesia, play therapists, and pediatric nursing, since it is likely children will represent a non-negligible proportion of their patient base. Third, during the continuing expansion of proton therapy use worldwide, pediatric medical and radiation oncologists need means for cooperation to share experience and expertise. The Pediatric Proton Consortium Registry presents one platform for this sharing but it is constrained by financial limitations and is currently only active in the U.S. [18]. Across oncology, pediatric solid tumors are rare. Collaboration on an international scale is necessary to improve evidence on the long-term benefits of protons compared to conformal photon therapy, both in terms of tumor control and long-term toxicities [9, 19]. While access to proton therapy remains limited in most countries, for the more consensual indications but especially for the many indications 
that remain debated, now is the ideal time to launch international collaborations to provide a high level of evidence (provided that methods and duration of follow-up are adequate) by comparing patient groups with similar clinical characteristics. Unfortunately, the current regulatory climate makes multi-site, multi-national research collaboration on clinical pediatric studies a challenge. Moreover, funding to coordinate high-quality, long-term clinical outcome studies needed for a comparative analysis of proton therapy has typically been viewed as low-priority in North America, Europe, and Asia. Until clinical evidence on long-term outcomes of proton therapy is available, decisions to refer pediatric patients for this treatment modality will continue to be based on dosimetric model comparisons, single institution experience, and/or expert recommendation, without a full knowledge on the possible late effects.

In conclusion, approximately 2,000 to 2,500 patients $<22$ years of age were treated with protons worldwide in 2016, mainly CNS tumors and axial sarcomas managed with curative intent but there was a wide variety of pediatric tumor types treated. Despite the limited data on late effects quantifying the value of protons compared to modern photon therapy techniques in pediatrics, a high consensus was observed across centers to consider rhabdomyosarcoma, medulloblastoma, ependymoma, lowgrade glioma, craniopharyngioma, intracranial germ-cell tumor, neuroblastoma and Ewing sarcoma as clinical indications for proton therapy, but only mild to low agreement remains for other tumor types. While the numbers of proton centers and clinical indications, especially for extra-cranial tumors, are rapidly increasing worldwide, there is urgent need and current opportunities to build international, observational studies on the late effects of protons and photons in pediatric patients. Assessing the long-term outcomes of proton therapy as well as of modern conformal photon techniques for tumor types that are currently considered both standard and non-standard indications for one or another technique in pediatrics is needed to provide evidence-based guidelines on which to base future treatment recommendations.

\section{REFERENCES}


[1] Glimelius B, Ask A, Bjelkengren G, Bjork-Eriksson T, Blomquist E, Johansson B, et al. Number of patients potentially eligible for proton therapy. Acta Oncol. 2005;44:836-49.

[2] International Agency for Research on Cancer (IARC). Tobacco Smoking In: Personal Habits and Indoor Combustions. IARC Monographs on the Evaluation of Carcinogenic Risks to Humans, Vol. 100E, IARC: Lyon, France; 2012; 1:43-212.

[3] Allard A, Haddy N, Le Deley MC, Rubino C, Lassalle M, Samsaldin A, et al. Role of radiation dose in the risk of secondary leukemia after a solid tumor in childhood treated between 1980 and 1999. Int J Radiat Oncol Biol Phys. 2010;78:1474-82.

[4] Allodji RS, Schwartz B, Veres C, Haddy N, Rubino C, Le Deley MC, et al. Risk of Subsequent Leukemia After a Solid Tumor in Childhood: Impact of Bone Marrow Radiation Therapy and Chemotherapy. Int J Radiat Oncol Biol Phys. 2015;93:658-67.

[5] Patel S, Kostaras X, Parliament M, Olivotto IA, Nordal R, Aronyk K, et al. Recommendations for the referral of patients for proton-beam therapy, an Alberta Health Services report: a model for Canada? Curr Oncol. 2014;21:251-62.

[6] Meattini I, Livi L, Saieva C, Marrazzo L, Rampini A, Iermano C, et al. Breast cancer following Hodgkin's Disease: the experience of the University of Florence. Breast J. 2010;16:290-6.

[7] Ladra MM, Edgington SK, Mahajan A, Grosshans D, Szymonifka J, Khan F, et al. A dosimetric comparison of proton and intensity modulated radiation therapy in pediatric rhabdomyosarcoma patients enrolled on a prospective phase II proton study. Radiother Oncol. 2014;113:77-83.

[8] Indelicato DJ, Merchant T, Laperriere N, Lassen Y, Vennarini S, Wolden S, et al. Consensus Report From the Stockholm Pediatric Proton Therapy Conference. Int J Radiat Oncol Biol Phys. 2016;96:387-92.

[9] Weber DC, Habrand JL, Hoppe BS, Hill Kayser C, Laack NN, Langendijk JA, et al. Proton therapy for pediatric malignancies: Fact, figures and costs. A joint consensus statement from the pediatric subcommittee of PTCOG, PROS and EPTN. Radiother Oncol. 2018.

[10] Ladra MM, Szymonifka JD, Mahajan A, Friedmann AM, Yong Yeap B, Goebel CP, et al. Preliminary results of a phase II trial of proton radiotherapy for pediatric rhabdomyosarcoma. J Clin Oncol. 2014;32:3762-70.

[11] Yock TI, Yeap BY, Ebb DH, Weyman E, Eaton BR, Sherry NA, et al. Long-term toxic effects of proton radiotherapy for paediatric medulloblastoma: a phase 2 single-arm study. Lancet Oncol. 2016;17:287-98.

[12] Leroy R, Benahmed N, Hulstaert F, Van Damme N, De Ruysscher D. Proton Therapy in Children: A Systematic Review of Clinical Effectiveness in 15 Pediatric Cancers. Int J Radiat Oncol Biol Phys. 2016;95:267-78. 
[13] Chung CS, Yock TI, Nelson K, Xu Y, Keating NL, Tarbell NJ. Incidence of second malignancies among patients treated with proton versus photon radiation. Int J Radiat Oncol Biol Phys. 2013;87:4652.

[14] Sethi RV, Shih HA, Yeap BY, Mouw KW, Petersen R, Kim DY, et al. Second nonocular tumors among survivors of retinoblastoma treated with contemporary photon and proton radiotherapy. Cancer. 2014;120:126-33.

[15] Pulsifer MB, Sethi RV, Kuhlthau KA, MacDonald SM, Tarbell NJ, Yock TI. Early Cognitive Outcomes Following Proton Radiation in Pediatric Patients With Brain and Central Nervous System Tumors. Int J Radiat Oncol Biol Phys. 2015;93:400-7.

[16] Mizumoto M, Murayama S, Akimoto T, Demizu Y, Fukushima T, Ishida Y, et al. Proton beam therapy for pediatric malignancies: a retrospective observational multicenter study in Japan. Cancer Med. 2016;5:1519-25.

[17] Chung CT, Bogart JA, Adams JF, Sagerman RH, Numann PJ, Tassiopoulos A, et al. Increased risk of breast cancer in splenectomized patients undergoing radiation therapy for Hodgkin's disease. Int J Radiat Oncol Biol Phys. 1997;37:405-9.

[18] Hess CB, Indelicato DJ, Paulino AC, Hartsell WF, Hill-Kayser CE, Perkins SM, et al. An Update From the Pediatric Proton Consortium Registry. Front Oncol. 2018;8:165.

[19] Berrington de Gonzalez A, Vikram B, Buchsbaum JC, de Vathaire F, Dorr W, Hass-Kogan D, et al. A Clarion Call for Large-Scale Collaborative Studies of Pediatric Proton Therapy. Int J Radiat Oncol Biol Phys. 2017;98:980-1.

[20] Chang AL, Yock TI, Mahajan A, Hill-Kaiser C, Keole S, Loredo L, et al. Pediatric Proton Therapy: Patterns of Care across the United States. Int J Particle Ther. 2014;1:357-67.

[21] Carrie C, Mahe MA. [Organization of the pediatric radiation oncology in France]. Cancer Radiother. 2009;13:525-6.

[22] Sakurai H, Ishikawa H, Okumura T. Proton beam therapy in Japan: current and future status. Jpn J Clin Oncol. 2016;46:885-92.

[23] Alapetite C, Timmermann B, Weber DC, Harrabi S, Ondrova B, Nilsson K, et al. Proton Beam Therapy for Children: Current Practice in Europe. In: Ther IJP, editor. 55th Annual Meeting of the Particle Therapy Cooperative Group (PTCOG). Prague, Czech Republic2016. p. 75.

[24] Knops RR, van Dalen EC, Mulder RL, Leclercq E, Knijnenburg SL, Kaspers GJ, et al. The volume effect in paediatric oncology: a systematic review. Ann Oncol. 2013;24:1749-53.

[25] Kann BH, Park HS, Lester-Coll NH, Yeboa DN, Benitez V, Khan AJ, et al. Postoperative Radiotherapy Patterns of Care and Survival Implications for Medulloblastoma in Young Children. JAMA Oncol. 2016;2:1574-81. 
[26] Geng C, Moteabbed M, Xie Y, Schuemann J, Yock T, Paganetti H. Assessing the radiationinduced second cancer risk in proton therapy for pediatric brain tumors: the impact of employing a patient-specific aperture in pencil beam scanning. Phys Med Biol. 2016;61:12-22.

[27] Paganetti H. Relative biological effectiveness (RBE) values for proton beam therapy. Variations as a function of biological endpoint, dose, and linear energy transfer. Phys Med Biol. 2014;59:R41972.

[28] Mohan R, Held KD, Story MD, Grosshans D, Capala J. Proceedings of the National Cancer Institute Workshop on Charged Particle Radiobiology. Int J Radiat Oncol Biol Phys. 2018;100:81631.

[29] Bonfrate A, Farah J, De Marzi L, Delacroix S, Herault J, Sayah R, et al. Influence of beam incidence and irradiation parameters on stray neutron doses to healthy organs of pediatric patients treated for an intracranial tumor with passive scattering proton therapy. Phys Med. 2016;32:590-9.

[30] Halg RA, Besserer J, Boschung M, Mayer S, Lomax AJ, Schneider U. Measurements of the neutron dose equivalent for various radiation qualities, treatment machines and delivery techniques in radiation therapy. Phys Med Biol. 2014;59:2457-68. 


\section{FIGURE CAPTIONS}

FIGURE 1. Number of pediatric patients treated in 2016 in 40 proton therapy centers according to the centers' first year operating

FIGURE 2. Proportion of proton centers treating each of the 15 most frequent tumor types in pediatric patients by continent

S-PNET: Supratentorial primitive neuroectodermal tumors; NGGC: non-germinomatous germ cell: *Tumors of the central nervous system. NB: Data are restricted to centers that treated $\geq 10$ pediatric patients (North America: $n=17$; Europe: $n=7$; Asia: $n=6$ ). The tumor types are sorted by descending order of total numbers of patients treated for each diagnosis in all countries. 
TABLE 1. Numbers of operating and participating centers in the survey, numbers of patients $<22$ years of age treated in 2016 and proportion of those patients living outside the treatment country, by continent and country

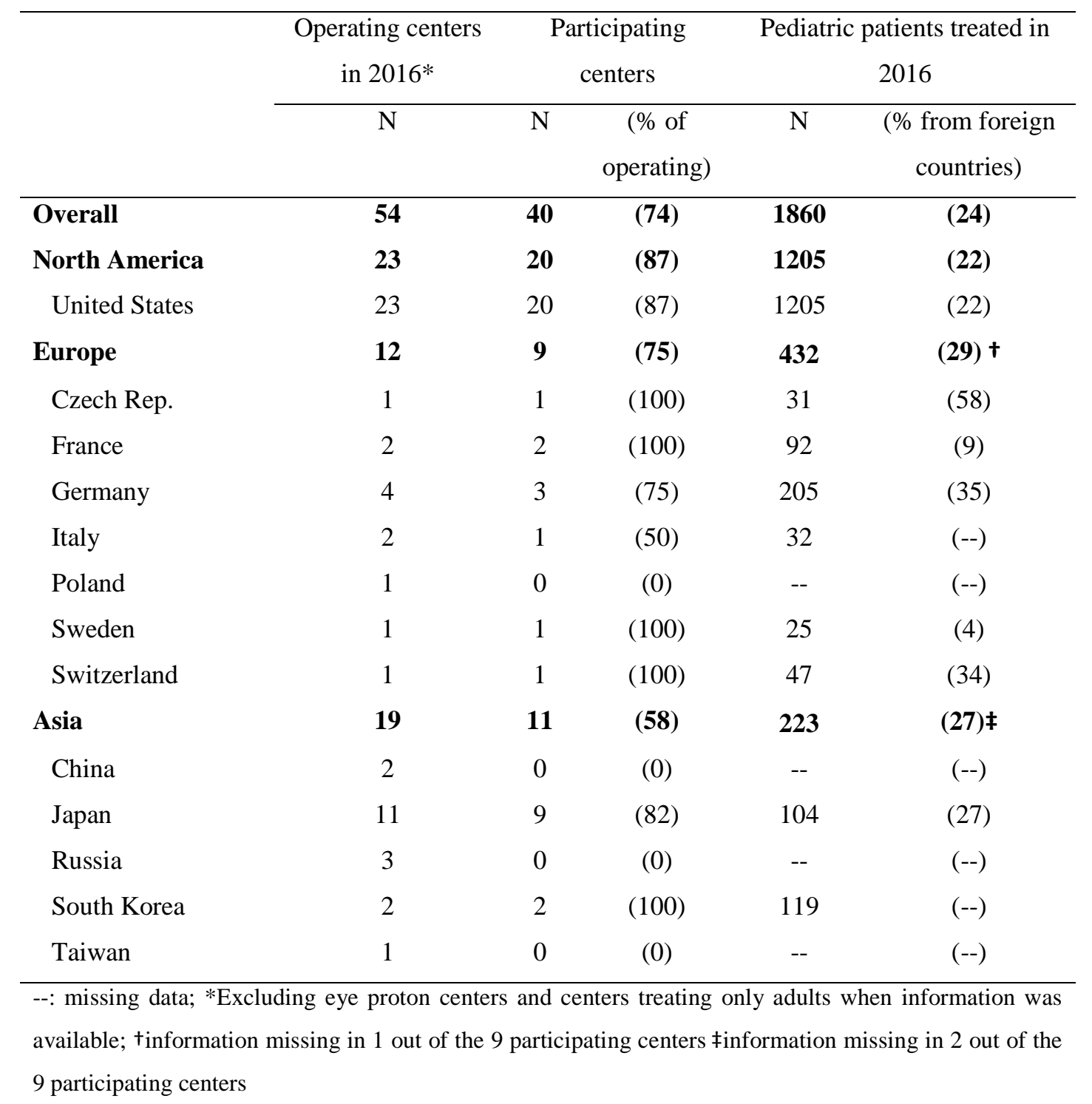


TABLE 2. Number $(\%)$ of patients $<22$ years of age according to the proton therapy delivery technique by continent and country

\begin{tabular}{lcccc}
\hline & $\begin{array}{c}\text { Passive } \\
\text { scattering beam }\end{array}$ & $\begin{array}{c}\text { Uniform } \\
\text { scanning beam }\end{array}$ & $\begin{array}{c}\text { Pencil beam } \\
\text { scanning }\end{array}$ & Unknown \\
\hline Overall & $\mathbf{6 2 5 ( 3 3 . 6 )}$ & $\mathbf{2 7 1 ( 1 4 . 6 )}$ & $\mathbf{9 4 7}(\mathbf{5 1 . 0})$ & $\mathbf{1 4 ( 0 . 8 )}$ \\
North America & $\mathbf{3 9 7 ( 3 2 . 9 )}$ & $\mathbf{1 8 2}(\mathbf{1 5 . 1})$ & $\mathbf{6 1 4}(\mathbf{5 1 . 0})$ & $\mathbf{1 2 ( 1 . 0 )}$ \\
United States & $397(32.9)$ & $182(15.1)$ & $614(51.0)$ & $12(1.0)$ \\
Europe & $\mathbf{1 1 7}(\mathbf{2 7 . 1})$ & $\mathbf{4 1 ( 9 . 5 )}$ & $\mathbf{2 7 4}(\mathbf{6 3 . 4})$ & $\mathbf{0}(\mathbf{0})$ \\
Czech Rep. & $0(0)$ & $0(0)$ & $31(100.0)$ & $0(0)$ \\
France & $90(97.8)$ & $0(0)$ & $2(2.2)$ & $0(0)$ \\
Germany & $27(13.2)$ & $41(20)$ & $137(66.8)$ & $0(0)$ \\
Italy & $0(0)$ & $0(0)$ & $32(100.0)$ & $0(0)$ \\
Sweden & $0(0)$ & $0(0)$ & $25(100.0)$ & $0(0)$ \\
Switzerland & $0(0)$ & $0(0)$ & $47(100.0)$ & $0(0)$ \\
Asia & $\mathbf{1 1 1}(\mathbf{4 9 . 8})$ & $\mathbf{4 8}(\mathbf{2 1 . 5})$ & $\mathbf{6 2}(\mathbf{2 7 . 8 )}$ & $\mathbf{2 ( 0 . 9 )}$ \\
Japan & $79(76.0)$ & $0(0)$ & $23(22.1)$ & $2(1.9)$ \\
South Korea & $32(26.9)$ & $48(40.3)$ & $39(32.8)$ & $0(0)$ \\
\hline
\end{tabular}


TABLE 3. Number (\%) of tumor types among patients $<22$ years of age treated with protons by continent and country

\begin{tabular}{|c|c|c|c|c|}
\hline & All countries & $\begin{array}{l}\text { North } \\
\text { America }\end{array}$ & Europe & Asia \\
\hline OVERALL & $1860(100.0)$ & $1205(100.0)$ & $432(100.0)$ & $223(100.0)$ \\
\hline CNS TUMORS & $890(47.8)$ & $562(46.6)$ & $246(56.9)$ & $82(36.8)$ \\
\hline Medulloblastoma & $233(12.5)$ & $149(12.4)$ & $59(13.7)$ & $25(11.2)$ \\
\hline Ependymoma & $208(11.2)$ & $131(10.9)$ & $62(14.4)$ & $15(6.7)$ \\
\hline Glioma, low grade* & $131(7.0)$ & $93(7.7)$ & $25(5.8)$ & $13(5.8)$ \\
\hline Craniopharyngioma & $101(5.4)$ & $61(5.1)$ & $34(7.9)$ & $6(2.7)$ \\
\hline Glioma, high grade ${ }^{\dagger}$ & $57(3.1)$ & $30(2.5)$ & $17(3.9)$ & $10(4.5)$ \\
\hline Atypical teratoid rhabdoid tumor & $50(2.7)$ & $28(2.3)$ & $19(4.4)$ & $3(1.3)$ \\
\hline Supratentorial primitive neuroectodermal tumors & $20(1.1)$ & $11(0.9)$ & $6(1.4)$ & $3(1.3)$ \\
\hline Pineoblastoma & $19(1.0)$ & $11(0.9)$ & $7(1.6)$ & $1(0.4)$ \\
\hline Meningioma & $17(0.9)$ & $14(1.2)$ & $2(0.5)$ & $1(0.4)$ \\
\hline Pituitary adenoma & $11(0.6)$ & $5(0.4)$ & $6(1.4)$ & $0(0)$ \\
\hline Choroid plexus carcinoma & $5(0.3)$ & $2(0.2)$ & $3(0.7)$ & $0(0)$ \\
\hline Pineal parenchymal tumor & $4(0.2)$ & $4(0.3)$ & $0(0)$ & $0(0)$ \\
\hline Other CNS tumors & $34(1.8)$ & $23(1.9)$ & $6(1.4)$ & $5(2.2)$ \\
\hline OTHER HEAD \& NECK DISEASES & $252(13.5)$ & $162(13.4)$ & $60(13.9)$ & $30(13.5)$ \\
\hline Skull base chordoma/chondrosarcoma & $42(2.3)$ & $25(2.1)$ & $16(3.7)$ & $1(0.4)$ \\
\hline Intracranial pure germinoma (intracranial) & $90(4.8)$ & $52(4.3)$ & $19(4.4)$ & $19(8.5)$ \\
\hline Non-germinomatous germ cell tumor (intracranial) & $31(1.7)$ & $25(2.1)$ & $3(0.7)$ & $3(1.3)$ \\
\hline Nasopharyngeal carcinoma & $22(1.2)$ & $15(1.2)$ & $5(1.2)$ & $2(0.9)$ \\
\hline Retinoblastoma & $17(0.9)$ & $8(0.7)$ & $6(1.4)$ & $3(1.3)$ \\
\hline Salivary gland tumors & $13(0.7)$ & $12(1.0)$ & $1(0.2)$ & $0(0)$ \\
\hline Arteriovenous malformation & $8(0.4)$ & $5(0.4)$ & $3(0.7)$ & $0(0)$ \\
\hline Hemangioma & $1(0.1)$ & $1(0.1)$ & $0(0)$ & $0(0)$ \\
\hline Uveal melanoma & $3(0.2)$ & $2(0.2)$ & $0(0)$ & $1(0.4)$ \\
\hline Other intracranial non-CNS tumors & $25(1.3)$ & $17(1.4)$ & $7(1.6)$ & $1(0.4)$ \\
\hline $\begin{array}{l}\text { NEUROBLASTOMA \& other peripheral nervous } \\
\text { cell tumors }\end{array}$ & $124(6.7)$ & $67(5.6)$ & $16(3.7)$ & $41(18.4)$ \\
\hline
\end{tabular}


Neuroblastoma

$\begin{array}{cccc}111(6.0) & 57(4.7) & 13(3.0) & 41(18.4) \\ 7(0.4) & 5(0.4) & 2(0.5) & 0(0) \\ 6(0.3) & 5(0.4) & 1(0.2) & 0(0)\end{array}$

Vestibular schwannoma

Esthesioneuroblastoma

\begin{tabular}{|c|c|c|c|}
\hline 467 (25.1) & 307 (25.5) & $104(24.1)$ & $56(25.1)$ \\
\hline 253 (13.6) & $162(13.4)$ & $52(12)$ & 39 (17.5) \\
\hline $128(6.9)$ & $91(7.6)$ & $27(6.3)$ & $10(4.5)$ \\
\hline $12(0.6)$ & $8(0.7)$ & $2(0.5)$ & $2(0.9)$ \\
\hline $10(0.5)$ & $5(0.4)$ & $3(0.7)$ & $2(0.9)$ \\
\hline $8(0.4)$ & $5(0.4)$ & $3(0.7)$ & $0(0)$ \\
\hline $56(3.0)$ & $36(3.0)$ & $17(3.9)$ & $3(1.3)$ \\
\hline $9(0.5)$ & 7 (0.6) & $1(0.2)$ & $1(0.4)$ \\
\hline $7(0.4)$ & $6(0.5)$ & $0(0)$ & $1(0.4)$ \\
\hline $2(0.1)$ & $1(0.1)$ & $1(0.2)$ & $0(0)$ \\
\hline $94(5.1)$ & $86(7.1)$ & $2(0.5)$ & $6(2.7)$ \\
\hline $80(4.3)$ & $77(6.4)$ & $1(0.2)$ & $2(0.9)$ \\
\hline $10(0.5)$ & $6(0.5)$ & $1(0.2)$ & $3(1.3)$ \\
\hline $3(0.2)$ & $2(0.2)$ & $0(0)$ & $1(0.4)$ \\
\hline $1(0.1)$ & $1(0.1)$ & $0(0)$ & $0(0)$ \\
\hline $24(1.3)$ & $14(1.2)$ & $3(0.7)$ & $7(3.1)$ \\
\hline
\end{tabular}

RR/UNSPECIFIED TUMORS

\section{CRANIAL)}

Rhabdomyosarcoma

Ewing sarcoma

Osteosarcoma

Non-skull base chordoma/chondrosarcoma

Desmoid tumor

Other or unknown sites/histology

UROGENITAL NEOPLASIA

Wilms tumor

Non-CNS germ cell tumor

\section{HEMATOPOETIC TUMORS}

Hodgkin Lymphoma

Non-Hodgkin Lymphoma

Leukemia

Other or unknown sites/histology

(EXTRA- 
TABLE 4. Estimated proportion of patients $<22$ years of age treated with protons for a malignant disease among all patients receiving radiotherapy in 2016 in the United States

\begin{tabular}{lcc}
\hline & $\begin{array}{c}\text { Projected number of } \\
\text { U.S. patients treated } \\
\text { with protons }\end{array}$ & $\begin{array}{c}\text { Proportion of all } \\
\text { patients receiving } \\
\text { radiotherapy }\end{array}$ \\
\hline Rhabdomyosarcoma & 145 & $54 \%$ \\
Medulloblastoma & 134 & $50 \%$ \\
Ependymoma* & 118 & $68 \%$ \\
Ewing Sarcoma & 82 & $53 \%$ \\
Neuroblastoma & 51 & $30 \%$ \\
Hodgkin lymphoma & 69 & $18 \%$ \\
Atypical teratoid rhabdoid tumor & 25 & $62 \%$ \\
Nasopharyngeal carcinoma & 13 & $46 \%$ \\
Primitive Neuroectodermal tumors & 10 & $17 \%$ \\
\hline
\end{tabular}

U.S.: United States; *Ependymoma cases include malignant and non-malignant subependymomas and myxopapillary ependymomas

NB: Numbers of U.S. patients treated with protons were projected after deducting the $22 \%$ of patients referred from abroad to be treated in a U.S. proton center, and assuming similar patterns of proton use in the 20 participating and the 3 non-participating centers in the 2016 survey. The numbers of all patients receiving radiotherapy were estimated based on 20142015 SEER data on cancer incidence and radiation treatment (released on April 2018). 


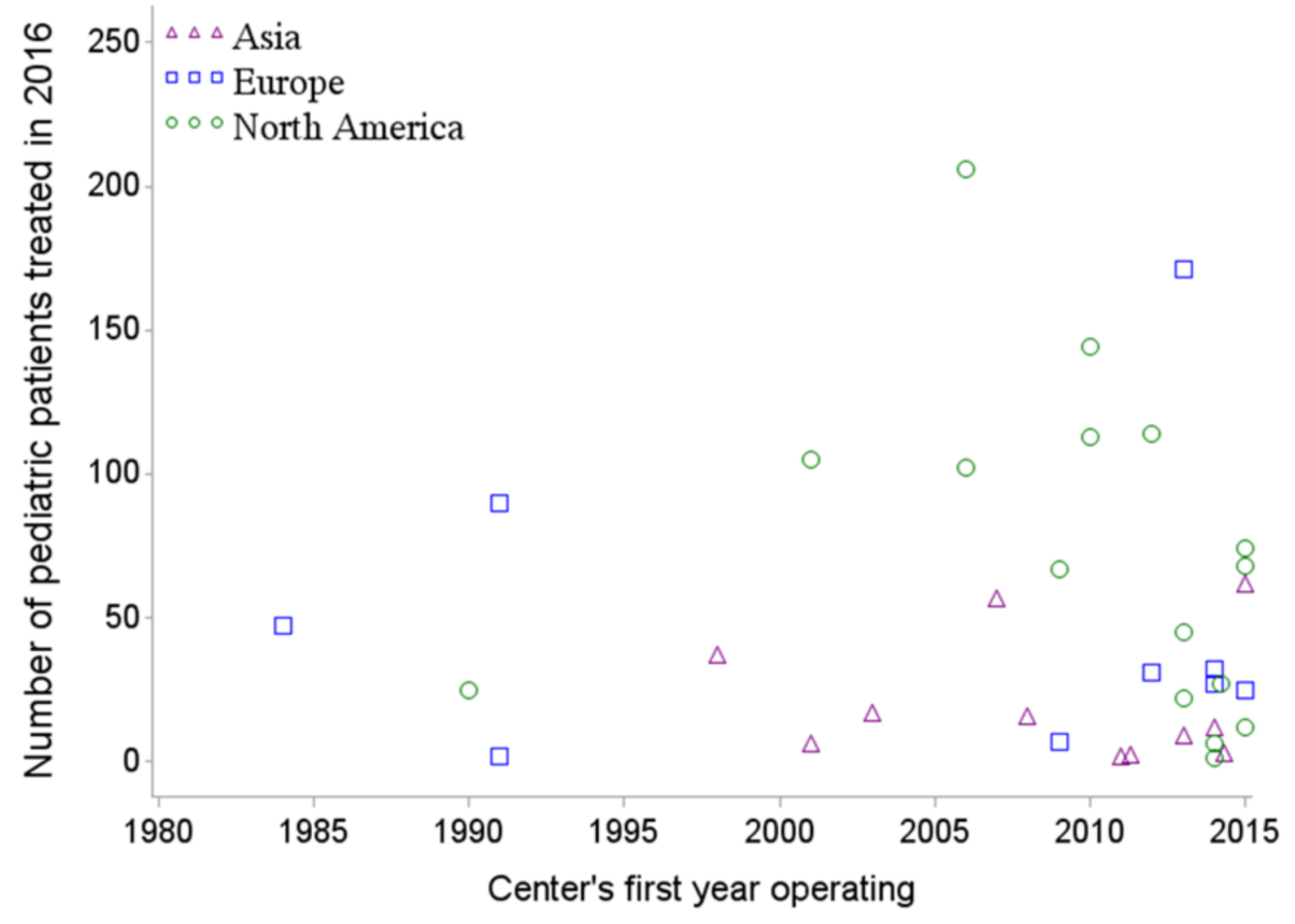


North America $\quad$ Europe $\quad \because$ Asia

Rhabdomyosarcoma

${ }^{*}$ Medulloblastoma

${ }^{*}$ Ependymoma

*Glioma (low grade)

Ewing sarcoma

Neuroblastoma

${ }^{*}$ Craniopharyngioma

Intracranial pure germinoma

Hodgkin Lymphoma

*Glioma (high grade)

${ }^{*}$ Atypical teratoid rhabdoid tumor

Skull base chordoma/chondrosarcoma

Intracranial NGGC tumor

Nasopharyngeal Carcinoma

*S-PNET

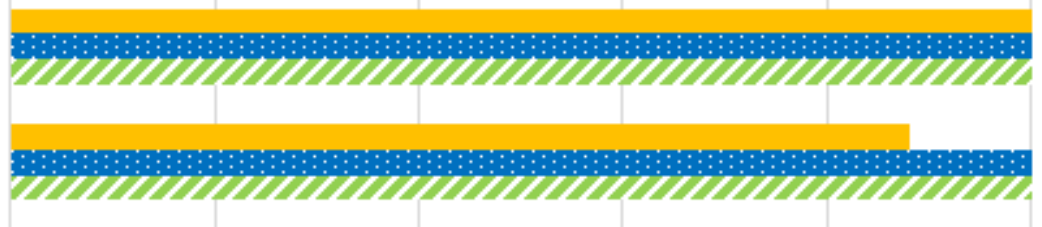

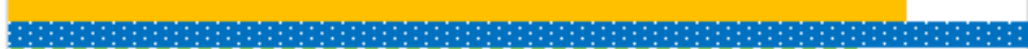

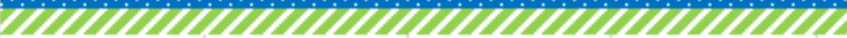

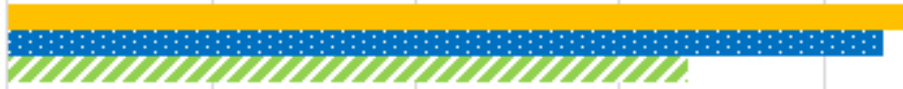

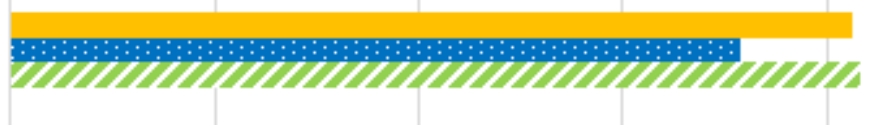

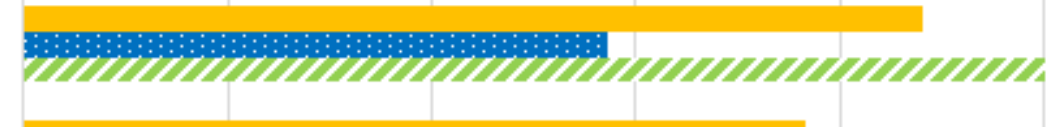
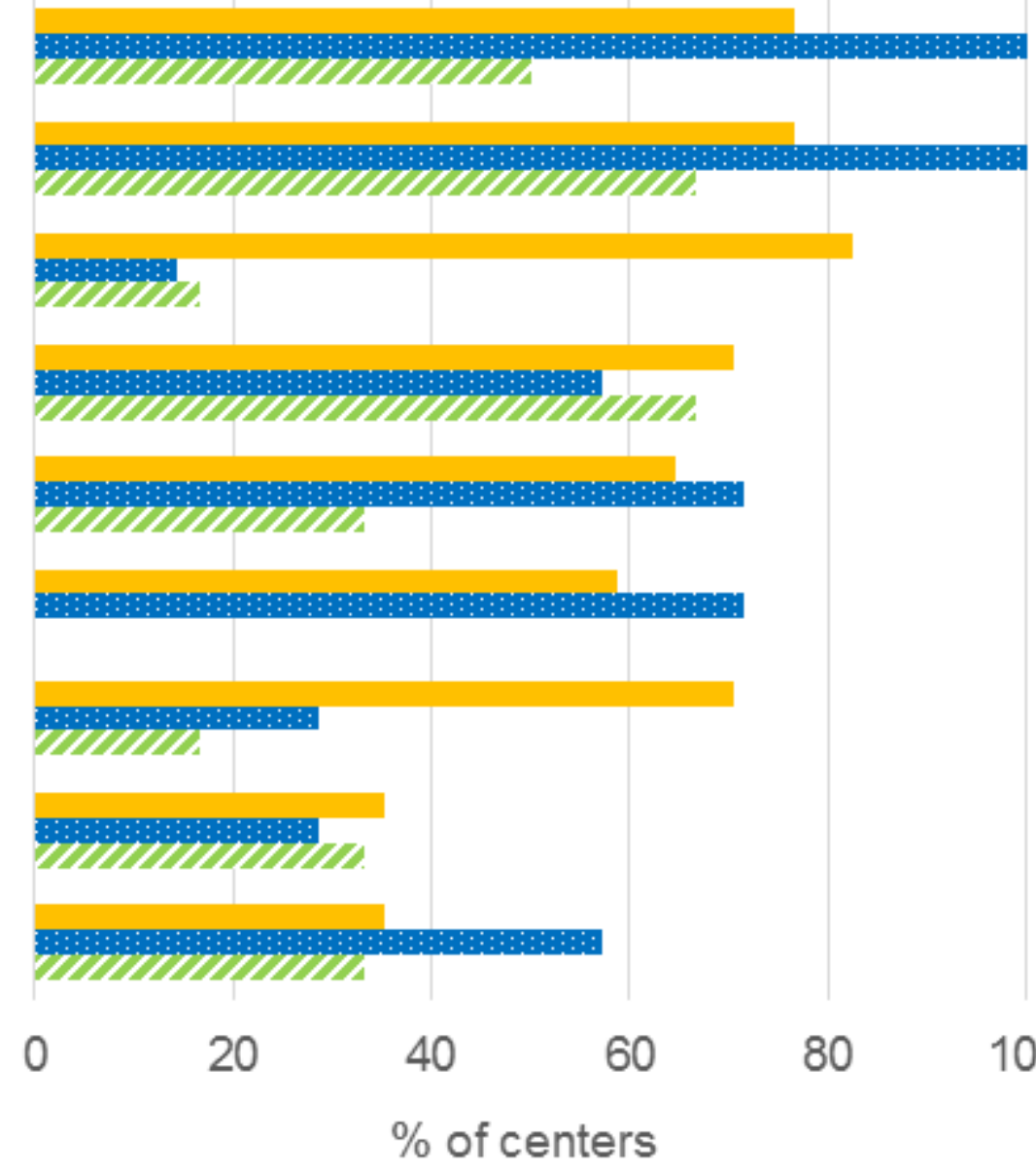

80

100

S-PNET: Supratentorial primitive neuroectodermal tumors; NGGC: non-germinomatous germ cell: *Tumors of the central nervous system. NB: Data are restricted to centers that treated $\geq 10$ pediatric patients (North America: $n=17$; Europe: $n=7$; Asia: $n=6$ ). The tumor types are sorted by descending order of total numbers of patients treated for each diagnosis in all countries. 